\title{
Depression and Anxiety Frequency in Patients Hospitalized on the Guadalajara Regional Military Hospital in the Month of April 2019
}

\author{
Miranda Nava Gabriel ${ }^{1 *}$ and Gallo Frías Luis Gilberto ${ }^{2}$ \\ ${ }^{1}$ Major of military armed forced of Mexico, Neurologist, Mexico \\ ${ }^{2}$ Medical Doctor, Social Service, Lamar University, Mexico
}

*Corresponding author: Miranda Nava Gabriel, Major of military armed forced of Mexico, Neurologist, Mexico

\begin{abstract}
Observe and Identify patients that presented depression and anxiety using the Hospital Anxiety Diagnosis Scale (HADS), Zung \& Conde scale and ASQ 15 scale on Guadalajara Regional Military Hospital during the month of April 01st to April 30th, 2019. Methods: This is a cohort, nonexperimental, observational, prospective and longitudinal study with PubMed and NCBI articles as variables. Findings: Most patients presented anxiety, most patients had a chronic illness, depression was seen mostly in patients older than 50 years old, there was inadequate diet, lack of sleep, and low distress level.
\end{abstract}

\section{Introduction}

Depression and anxiety affect most people around the world, it is characterized by a presence of fear, loss of interest, feelings of guilt or self-esteem that are more commonly associated with sleep disorders, lack of appetite, lack of energy or difficulty concentrating. Depression can become chronic or recurrent and difficult the overall performance on a daily basis, or capacity to live day by day, in its most dangerous form it can lead often to suicide and its lowest form it can be treated with medication and professional psychotherapy [1]. Anxiety is one of the major disorders and its characterized by persistent concern during any activity or routine it is difficult to treat, and it can affect the way a person feels physically [2]. During this investigation we will observe a sample that was taken on the Guadalajara Regional Military Hospital during the month of April a sample of 56 patients presented anxiety and depression according to three scales that were applied.

\section{HADS (hospital anxiety diagnosis scale)}

The Hospital Anxiety Diagnosis Scale is an auto applicable questionnaire integrated by 14 items with subscales of seven items one for impared questions and one with pair questions for depression, the authors for this scale are Zigmund and Snaith who proposed this in 1983 and defined the concepts of anxiety and depression the objective of this scale is to identify if the patient has being tensed, concerned or frightened in any way, the 8 items that form the depression subscale are centered around anhedonia with a maximum score that binds from 0 to a 39 score, in which 0-9 score means lack of stress, 10-19 means low stress, 19 to 29 means mild stress and 30 to 39 means anxiety and severe depression.

Zung \& conde scale: Its and auto applicable scale consisting of 20 phrases related to depression formed by 10 negative phrases and 10 positive phrases which relate to strong somatic symptoms and 8 cognitive items for each group contemplating the scale with two items referee to mood and other psychotic symptoms [3].

Depression and anxiety: Severe Depression: Its characterized by a combination of symptoms that interfere with capacity to work, sleep, study, eat and enjoy daily basis activities.

Dysthymic disorder: Its characterized by symptoms that is somewhat between 2 years and beyond but less severe, it incapacitates the patient and it prevents him from having a normal life accompanied by a severe depression episode during life [4-6]. 
Psychotic depression: Occurs during severe depression and its accompanied by some form of psychosis accompanied by delirium and hallucinations.

Seasonal Depression: Its characterized by depression that appears during Winter or times of decreased sunlight.

Bipolar Disorder: Its characterized by maniac depression disorder that its accompanied by cyclic mood swings and depression state, its often seen in patients with cancer, HIV/Aids and Parkinson.

\section{Symptoms}

\section{Emotional}

Are accompanied by guilt ideas, a severe disease, ideas of sadness never going to heal, loneliness, lack of concentration because patient will eventually die.

\section{Physical}

Difficulty eating, or basic needs, weight loss, mood swings.

\section{Negative thoughts}

This is mostly seen in older patients, self-stem problems, most cases are seen in patients over 60 years old, or below 45 years old.

\section{Methods}

This is a cohort, non-experimental, observational, prospective and longitudinal study in which scholarity was evaluated, cause of hospitalization, age, previous diseases, job and the days patient had been hospitalized.

56 patients both men and women older tan 18 years old were evaluated during this study, a random sample was taken in which every patient has the same possibility of presenting depression or anxiety [6-8]. Patients hospitalized in the women's hospital room, the men's hospital room, and the room that consisted of patients that had the rank of major in the Mexican armed forces or above excluding patients that belonged to Intensive care unit, using the Hospital Anxiety Diagnosis Scale, Zung \& Conde Scale and ASQ-15 Scale were used during this study (Figure 1-3) [9,10].

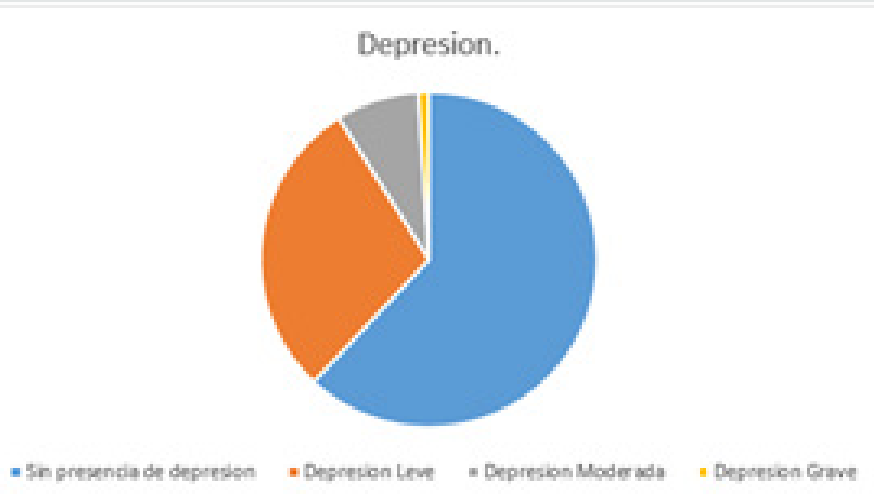

Figure 1: Patients with depression. Fuente. Zung \& Conde Depression Scale. $62 \%$ of the patients did not present depression 34 patients, 16 patients had low depression $29 \%$, also $8 \%$ of the patients had moderate depression which represented 4 patients also 2 of the patients representing 1\% presented what could be considered as severe depression

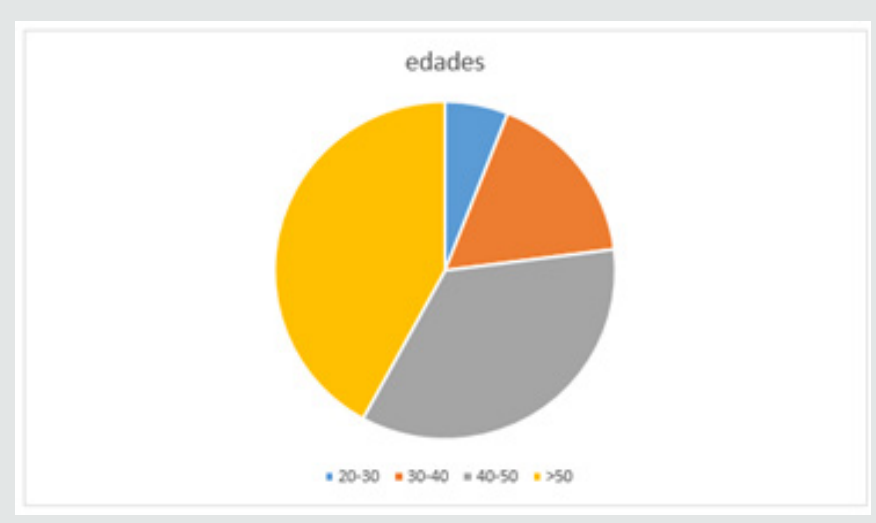

Figure 2: Most common ages seen during study. $42 \%$ of the patients presented depression were over 50 years old. $35 \%$ of the patients presented depression between $40-50$ years of age. $17 \%$ of the patients that presented depression had between $30-40$ years of age. $6 \%$ of the patients had between the ages of 20 to 30 years of age. 


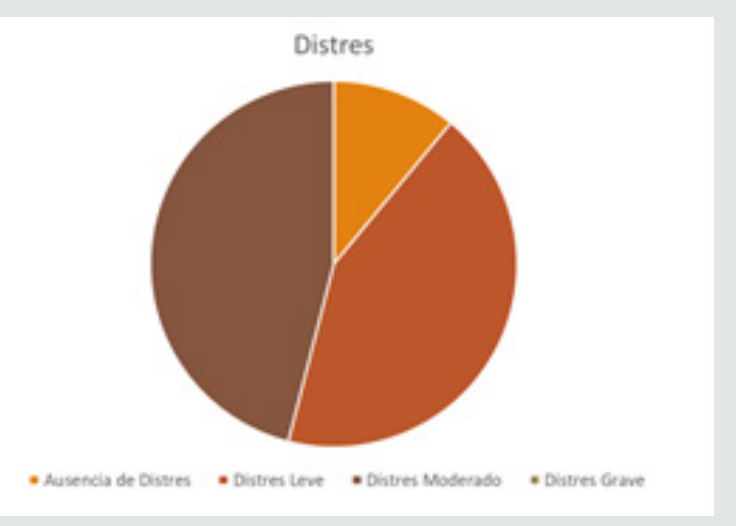

Figure 3: Patients that had some sort of stress evaluated by HADS scale.

a) $\quad 43 \%$ of the patients had lack of stress.

b) $46 \%$ of the patients had low level stress.

c) $11 \%$ of the patients had moderate stress.

d) $0 \%$ of the patients had severe stress

\section{Justification}

This study was conducted to observe what was the impact of being hospitalized and the relation it had with depression and anxiety in patient, we pretended to find viable date that allowed us to expose the hospital environment and the presence of disease, anxiety and depression (Figure 4) [11,12].

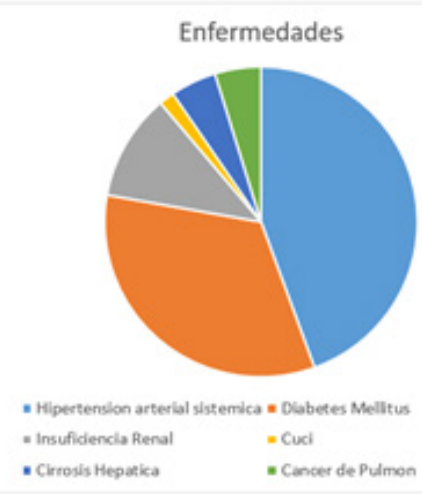

Figure 4: Most common diseases (12 patients didn't have an illness):

a) Hypertension: 16

b) Diabetes Mellitus:12

c) Renal Insufficiency: 4

d) Ulcerative Colitis: 1

e) Hepatic Cirrosis: 3

f) Lung Cancer: 3

g) Cervical Cancer:1

h) Fractures: 4

\section{Results}

Figure 1 Patients with depression. Fuente. Zung \& Conde Depression Scale. $62 \%$ of the patients did not present depression 34 patients, 16 patients had low depression $29 \%$, also $8 \%$ of the patients had moderate depression which represented 4 patients also 2 of the patients representing 1\% presented what could be considered as severe depression (Figure 5,6). 


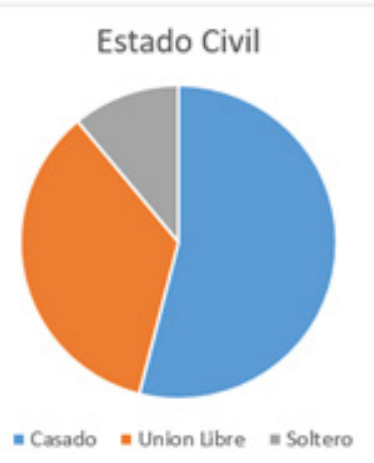

Figure 5: Civil Status:

a) Married: $54 \%$

b) Separated: $35 \%$

c) Single: $11 \%$

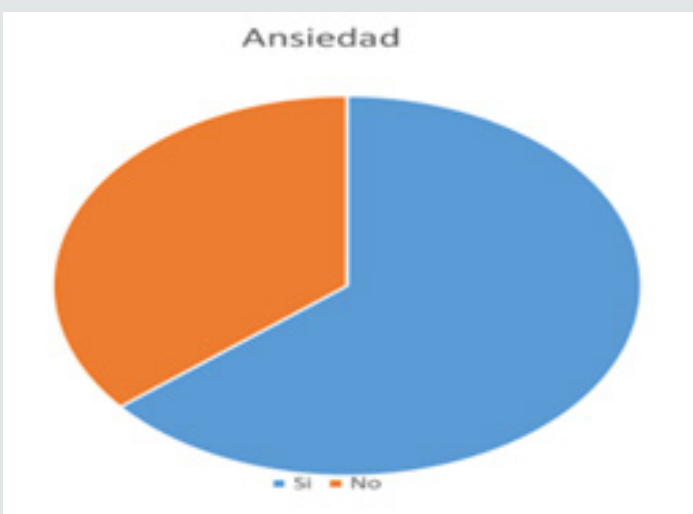

Figure 6: Patients that presented anxiety

a) $64 \%$ presented anxiety 35 patients

b) $36 \%$ did not present anxiety 21 patients

c) $86 \% \mathrm{No}$

d) $14 \%$ Yes

\section{Conclusion}

Referred to this subject we understand this two disorders are preventable and the patient if its treated and seeks help with time he can change his daily habits, our suggestions are that a stable lifestyle with a well-balanced diet consisting of fruit, vegetables, meat, daily exercise, stable relationships with family and friends, alongside no work stress, in addition to a good mental stability can lead to a good life and to prevent this type of disorders also to prevent chronic illness which were seen during this study on most patients that indicated feeling anxious or depressed, if they are in this state also to take medication on time and with the help of family members and friend. a) We observe most patients presented a low depression level

b) Most patients presented anxiety

c) Most patients had a chronic illness

d) Patients over 50 years old presented higher depression levels

e) Most patients were married

Most patients had low level stress level.

\section{References}

1. Carolina Casanova (2017) Screening for symptoms of anxiety and depression in patients admitted to a university hospital with acute coronary syndrome. Trends in Psychiatry and Psychotherapy 39(1): 1218. 
2. CPG (2008) Clinical Practice Guideline Working Group for the management of patients with anxiety disorders in primary care. National Plan for SNS, Clinical practice guidelines in SNS.

3. Psicodex (2016) Psychiatry and Psychology Service, Dexeus University Hospital Barcelona, Spain.

4. National Institute of Mental Health. Department of Health and Human Services. Anxiety disorders, United States.

5. Fernando A Wagner, Catalina Gonzalez (2012) Focusing depression as a public health problem in Mexico. Menta Health 35(1).

6. Guillermo Hernández G, Gricel Orellana V, Mónica Kimelman J, Carlos Nuñez M, Carolina Ibáñez H (2005) Anxiety disorders in patients hospitalized in Internal Medicine anxiety disorders among patients hospitalized in a medical ward. Rev Méd Chile 133: 895-902.
7. Miriam Costas, Veronica Prado, Jose Manuel Crespo (2013) Anxiety and depression among hospitalized patients in the Ferrol hospital complex.

8. Sílvia Abduch Haas, Daniela Centenaro Levandowski, Antônio Nocchi Kalil (2017) Anxiety, depression, perception and feelings of women with cancer indicated for pelvic exenteration surgery.

9. Ye Li, Mei RongLv, Yan Jin Wei, Ling Sun, Ji Xiang Zhang (2017) Dietary patterns and depression risk: A meta-analysis. Psychiatry Research 253: 373-382.

10. General IMSS. Health Council Quick Reference Guide Practical Guide.

11. World Health Organization. What is depression and anxiety? Geneva, Switzerland.

12. Roza M (2019) self-administered psychomag depression scale. Zung and Count scale that is.
(C) This work is licensed under Creative

To Submit Your Article Click Here: Submit Article

DOI: $10.32474 / 0 J N B D .2020 .03 .000171$

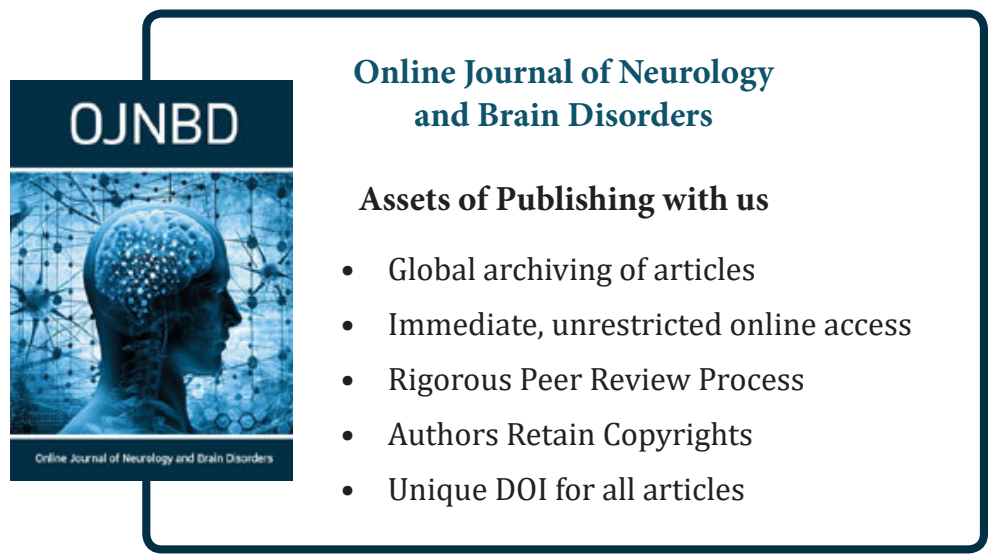

